\title{
Successful percutaneous coronary intervention in patients with recanalized thrombus: Saving a radial artery by snuffbox approach
}

\author{
Yongcheol Kim, Myung Ho Jeong, Min Chul Kim, Doo Sun Sim, \\ Young Joon Hong, Ju Han Kim, Youngkeun Ahn \\ Department of Cardiology, Chonnam National University Hospital, Gwangju, Korea
}

A 76-year-old man with chronic kidney disease was referred to the documented clinic suffering from aggravating effort angina. Thus, after treatment with acetylsalicylic acid (300 mg loading dose) and clopidogrel (300 mg loading dose), coronary angiography was tried via left distal radial artery as a preservation of radial artery for arteriovenous fistula creation. This was successfully cannulated by a 5 French sheath (Fig. 1). However,

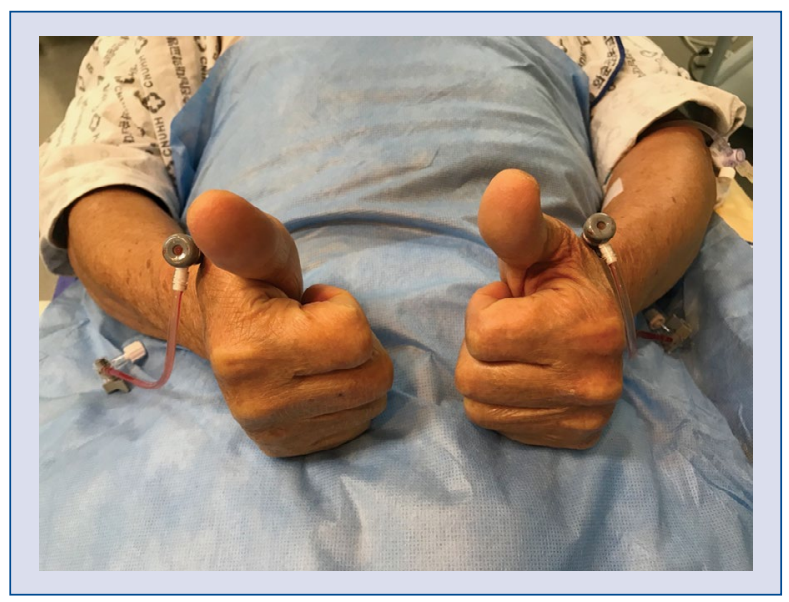

Figure 1. Inserted 5 French sheath via snuffbox approach. brachial artery anomaly led to a change in the access site using the right snuffbox approach (Fig. 2A). Coronary angiography demonstrated diffuse stenosis with multiple linear filling defects and haziness in the proximal right coronary artery (Fig. 2B). Optical coherence tomography (OCT) showed a honeycomb-like structure with multiple cavities and it was concluded that this represented recanalized thrombus (Fig. 2C, D, Suppl. Video 1). OCT assessment led to implantation of a $2.75 \times$ $\times 38 \mathrm{~mm}$ Xience Sierra stent (Abbott Vascular, Santa Clara, CA, USA) and postdilation was achieved with a $3.25 \times 12 \mathrm{~mm}$ noncompliant at up to $21 \mathrm{~atm}$ in the proximal portion of the implanted stent. Repeated OCT assessment demonstrated good stent expansion and strut apposition without edge dissection. Final coronary angiography showed good distal flow without residual stenosis (Fig. 2E).

This case highlights that OCT enables confirmation of a rare case of recanalized thrombus and the snuffbox approach can be an alternative access site in patients with renal impairment where the radial artery needs protection for anteriovenous fistula.

Conflict of interest: None declared

\footnotetext{
Address for correspondence: Myung Ho Jeong, MD, Cardiovascular Convergence Research Center of Chonnam National University Hospital, Gwangju 501-757, Republic of Korea, tel: +82-62-220-6243, fax: +82-62-228-7174, e-mail: myungho@chollian.net
}

Received: 30.11.2018 Accepted: 21.01.2019 


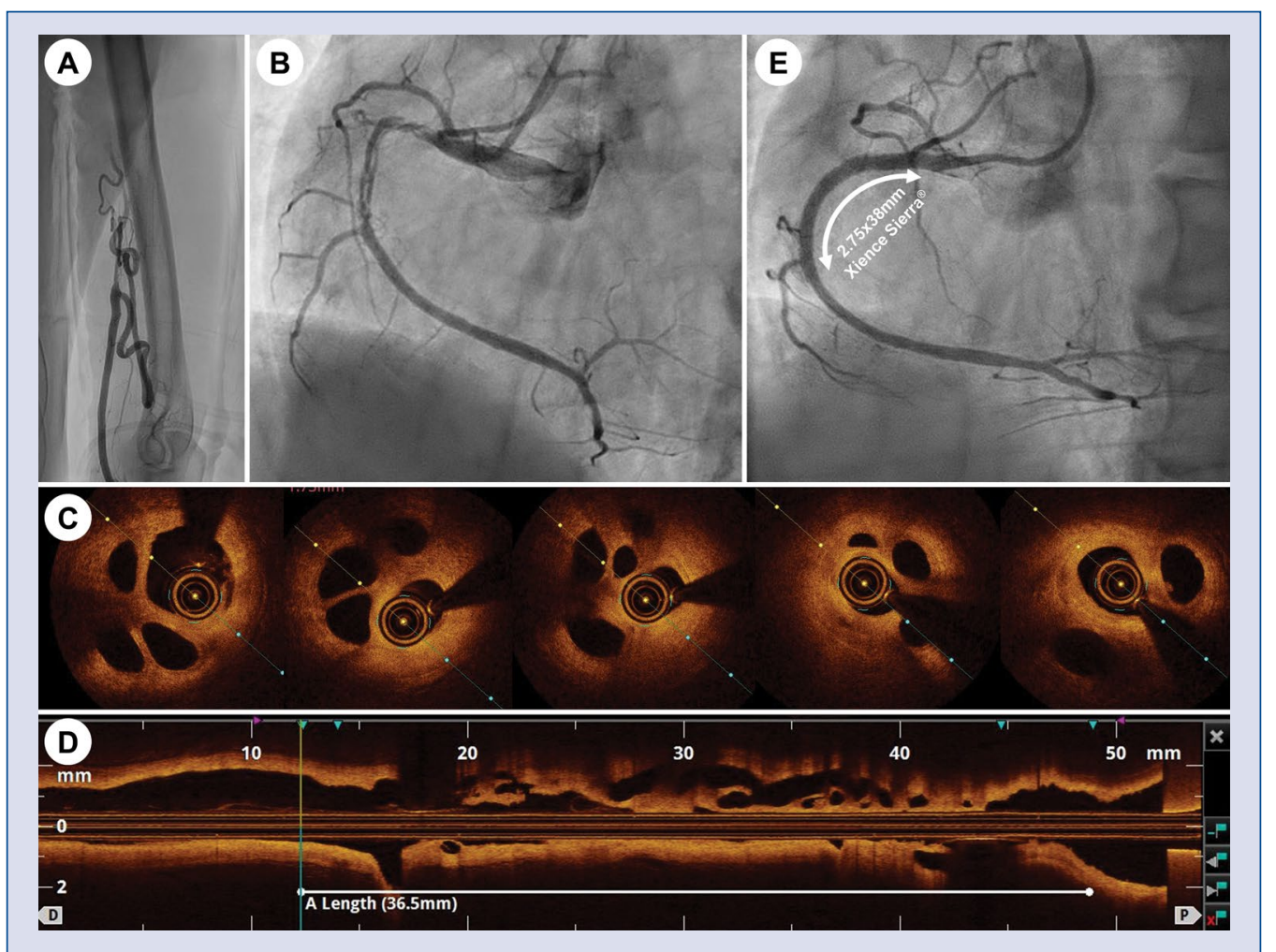

Figure 2. A. Peripheral angiography demonstrating the left brachial artery anomaly; B. Angiographic assessment demonstrating multiple linear filling defects and haziness in the proximal coronary artery; C, D. Longitudinal and cross-sectional optical coherence tomography imaging demonstrated a honeycomb-like structure with multiple cavities of various sizes; E. Final angiogram demonstrated good distal flow without residual stenosis from treatment with $2.75 \times 38 \mathrm{~mm}$ Xience Sierra ${ }^{\circledR}$ stent. 